QUIPUKAMAYOC | Revista de la Facultad de Ciencias Contables

Vol. $16 \mathrm{~N}^{\circ}$ 32, pp. 57-64 (2009) UNMSM, Lima - Perú

ISSN: 1560-9103 (versión impresa) / ISSN: 1609-8196 (versión electrónica)

\title{
EL XBRL, NUEVO LENGUAJE DE COMUNICACION DE LOS CONTADORES PUBLICOS DEL PERU
}

\author{
THE XBRL, NEW LANGUAGUE OF COMMUNICATION OF \\ PUBLIC ACCOUNTANTS OF PERU
}

\author{
Carlos Alberto Pastor Carrasco ${ }^{1}$ \\ Docente Asociado de la Facultad de Ciencias Contables, UNMSM
}

(Recepción: Agosto de 2009 / Conformidad: Setiembre de 2009)

"La Comunicación es irreversible, transformable e inevitable."

Anónimo

\section{RESUMEN}

Las siglas XBRL significan: eXtensible Business Reporting Language. Es un lenguaje más de la familia de «XML ${ }^{2} »$ que se está convirtiendo en un estándar de los medios de comunicación de información entre las empresas en Internet. Es un lenguaje para la comunicación electrónica de datos financieros y de negocios que está revolucionando el modo de informar en las empresas de todo el mundo. Proporciona beneficios importantes en la preparación, análisis y comunicación de información empresarial. Ofrece ahorro de costos, mayor eficiencia y mejor precisión y fiabilidad a todos los implicados en el suministro o en el uso de los datos financieros.

El profesional contable es muy consciente de la necesidad de sistematizar el intercambio de información financiera a consecuencia de sus muchos años de experiencia con sistemas contables automatizados. En poco más de 11 años esta sencilla idea ha conseguido atraer el interés y el respaldo de toda una comunidad de individuos y organizaciones públicas y privadas que representan un gran número de países, coordinados en la XBRL Internacional, una organización internacional sin ánimo de lucro, que lidera el desarrollo y mantenimiento del estándar XBRL y a la que pertenecen más de 550 instituciones.

Se investiga el tema del estándar XBRL con la finalidad de fomentar su difusión y futuro uso por parte de los contadores públicos del país a fin de facilitar el intercambio de información financiera entre las empresas y el gobierno. Asimismo, busca incentivar entre los alumnos de la profesión contable el desarrollo de proyectos de investigación que permitan la creación de la taxonomía necesaria para su utilización.

Palabras Claves: Comunicación, Informes Contables, XBRL, XML

\section{ABSTRACT}

The initials XBRL means: eXpansible Business Reporting Language. It is a language more than the family of $\mathrm{XML}^{2}$ that is transforming into a standard of the media of information among the companies in Internet. It is a language for the electronic communication of financial data and of businesses that it is revolutionizing the way of informing from all over the world in the companies. It provides important benefits in the preparation, analysis and communication of managerial information. He offers saving of costs, bigger efficiency and better precision and reliability to all those implied in the supply or in the use of the financial data.

The countable professional is very aware of the necessity of systematizing the exchange of financial information as a consequence of her many years of experience with countable automated systems. In little more than 11 years this simple idea has been able to attract the interest and the back of an entire community of individuals and public and private organizations that represent a great number of countries, coordinated in International XBRL, an international organization without spirit of lucre that leads the development and maintenance of standard XBRL and to the one that they belong more than 550 institutions.

The topic of standard XBRL is investigated with the purpose of fomenting its diffusion and future use on the part of the public accountants of the country in order to facilitate the exchange of financial information between the companies and the government. Also, it looks for to motivate among the students of the countable profession the development of investigation projects that they allow the creation of the necessary taxonomy for their use.

Key words: Communication, Countable Reports, XBRL, XML

\footnotetext{
Contador Público Colegiado Certificado. Docente Investigador de la Facultad de Ciencias Contables, UNMSM. E-mail: cpc5637@hotmail.com

2 Extensible Markup Language (Lenguaje de Marcas Extensible)
} 


\section{INTRODUCCIÓN}

En los últimos 20 años se ha producido un cambio del mundo analógico al mundo digital, habiéndose dotado de la característica de ubicuidad a la información, al hacerla accesible desde cualquier parte del planeta, reduciendo al mismo tiempo los costos, tanto de su generación como de su difusión y almacenamiento.

Con el empleo de la computadora en los negocios, la capacidad para crear información sobre la situación de sus negocios ha crecido, así como las posibilidades de difundirla con un costo reducido, se materializaron con la generalización del uso de la red Internet como vehículo de intercambio. Quienes demandan información (entre otros, los inversores, prestamistas y acreedores, la administración tributaria, los empleados y las unidades estadísticas y de análisis) disponen de una base de datos creciente, que permite el almacenamiento de la ingente producción de información económico-financiera que emiten las unidades analizadas.

A pesar de la enorme capacidad para generar y recibir información, los dos polos de la comunicación (las empresas, y los que requieran de su información) se muestran insatisfechos con la situación actual, porque además tienen que asumir el costo de los procesos de captura y depuración de la información y no la reciben en el momento en el que la precisan, ni con la periodicidad requerida, ni con el nivel de detalle deseado, habría que añadir el costo derivado del riesgo de error en el que se incurre en la fase de grabación de los datos.

Las Empresas, las entidades supervisoras y reguladoras de nuestro País, así como las del Exterior, vienen demandando que la información y gestión financiera sea transparente, precisa, de calidad y que sea emitida en periodos de tiempo muy reducidos. El Acuerdo de Basilea II y las Normas Internacionales de Contabilidad son dos regulaciones que van a cambiar la gestión financiera de las empresas.

El XBRL (eXtensible Business Reporting Language - Lenguaje extensible de informes de negocios) es el estándar que permite unificar el tratamiento de la información financiera bajo un único formato, que servirá para adaptarnos a la economía del siglo XXI.
Queremos ser parte de ese proceso, trabajando para implementar en las empresas el XBRL. Debemos estar concientes de cual es el riesgo y la oportunidad perdida de no publicar los informes contables en XBRL.

\section{ANTECEDENTES}

EL XBRL proviene del lenguaje XML- Extensible Markup Language (Lenguaje de Marcas Extensibles) y éste a su vez proviene de un lenguaje inventado por IBM en los años 70, llamado GML (Generalized Markup Language), el cual surgió por la necesidad que tenía la empresa IBM de almacenar grandes cantidades de información propia y de sus clientes. Este lenguaje gustó mucho a la ISO ${ }^{2}$, motivo por el cual en el año 1986 trabajaron conjuntamente para normalizarlo, creando así el lenguaje denominado SGML (Standard Generalized Markup Language) ${ }^{3}$, éste fue capaz de adaptarse a un gran abanico de problemas existentes. A partir de este lenguaje se han creado otros sistemas que permiten almacenar grandes volúmenes de información.

En el año 1989 Tim Berners Lee creó la web ${ }^{4}$, y junto con ella el lenguaje HTML $^{5}$. Este lenguaje se definió en el marco de SGML y fue la aplicación más conocida de este estándar. Los navegadores web; sin embargo, siempre han puesto pocas exigencias al código HTML que interpretan y así las páginas web son caóticas y no cumplen con la sintaxis. Estas páginas web dependen fuertemente de una forma específica de lidiar con los errores y las ambigüedades, lo que hace a las páginas más frágiles y a los navegadores más complejos.

Otra limitación del HTML es que cada documento pertenece a un vocabulario fijo, que es establecido por el DTD ${ }^{6}$. No se pueden combinar elementos de diferentes vocabularios. Asimismo es imposible para un intérprete (por ejemplo un navegador) analizar el documento sin tener conocimiento de su gramática (del DTD).

E1 XML, es un metalenguaje extensible de etiquetas desarrollado por el World Wide Web Consortium (W3C) ${ }^{7}$. Es una simplificación y adaptación del SGML y permite definir la gramática de lenguajes específicos (de la misma manera que HTML es a su vez un lenguaje definido por SGML).

\footnotetext{
International Organization for Stardardization (Organización Internacional para la Estandarización)

Norma ISO 8879:1986

World Wide Web, cuya traducción podría ser Red Global Mundial

Hyper'Text Markup Language (Lenguaje de Marcas de Hipertexto)

Document Type Definition (Definición de Tipo de Documento)

http://www.w3.org/
} 


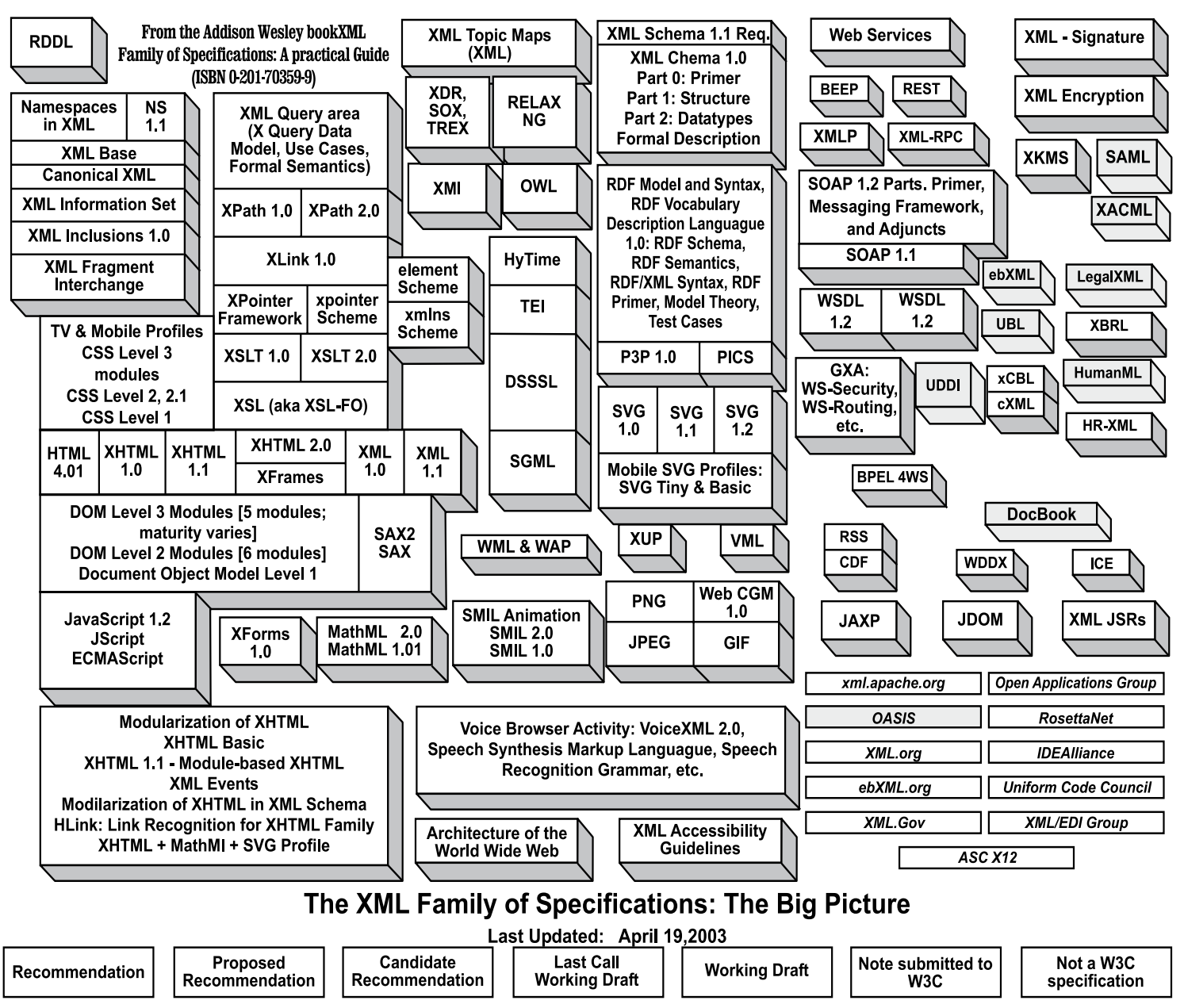

Copyright (c) 1998-2003 Kenneth B. Sall. All Rights Reserved. http://kensall.com/big-picture/

\section{Ilustración 1 La familia XML}

Por lo tanto XML no es realmente un lenguaje en particular, sino una manera de definir lenguajes para diferentes necesidades (Ver Ilustración 1 La familia $\mathrm{XML}$ ). Algunos de estos lenguajes que usan XML para su definición son XHTML, SVG, MathML. ${ }^{8}$

Se crea el XML no sólo para su aplicación en Internet, sino que se propone como un estándar para el intercambio de información estructurada entre diferentes plataformas. Es permitido usarse en bases de datos, editores de texto, hojas de cálculo y casi cualquier cosa imaginable.

XML es una tecnología sencilla que tiene a su alrededor otras que la complementan y la hacen mucho más grande y con unas posibilidades mucho mayores. Tiene un papel muy importante en la actualidad ya que permite la compatibilidad entre sistemas para compartir la información de una manera segura, fiable y fácil.

A nivel mundial, en la mayoría de los países existe una mayor tendencia a la implantación del standard XBRL. Principalmente en Estados Unidos y Japón, mientras que a nivel de Europa destacan Alemania y el Reino Unido. Fabricantes, como Software AG o Microsoft, ya disponen en su catálogo de aplicaciones que permiten realizar informes en XBRL. España es uno de los países de habla hispana que difunden el XBRL a través de su portal http://www.xbrl.es/ 

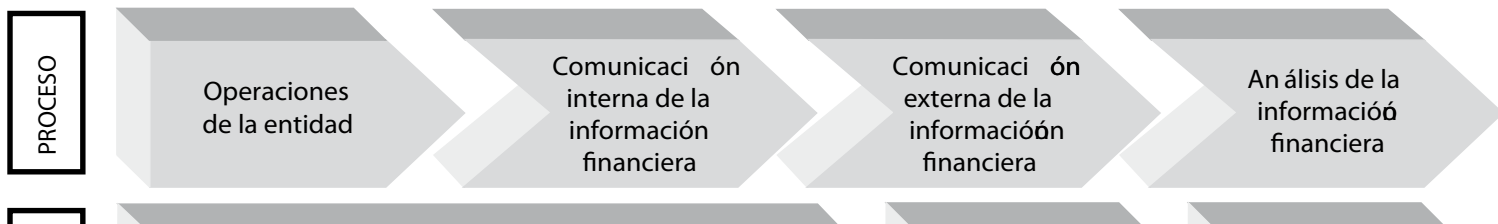

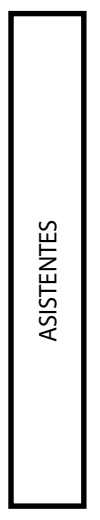
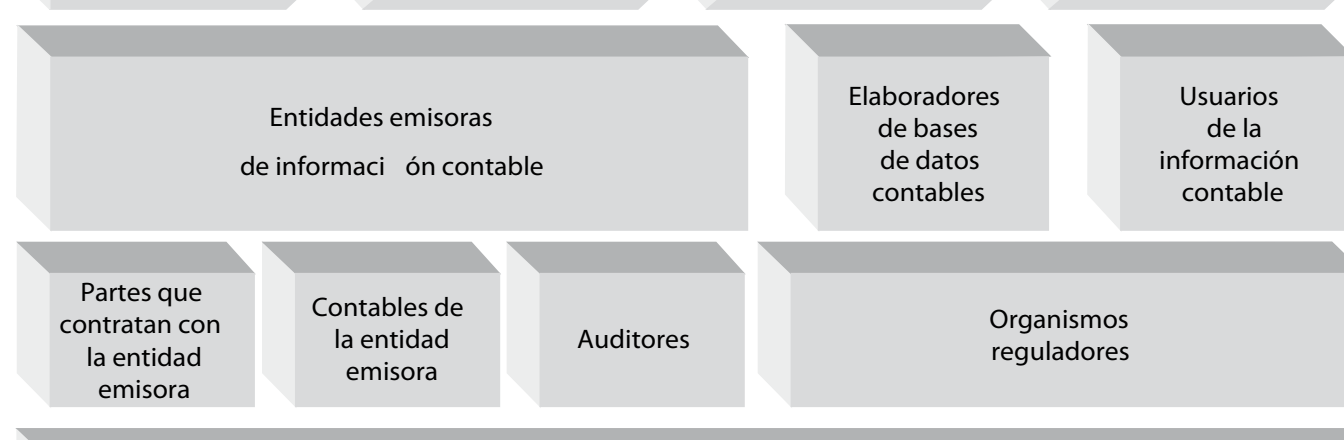

Desarrolladores de programas inform áticos para la elaboraci ón y el an álisis de la informaci ón contabl e

\section{llustración 2 Reporting Financiero}

\section{Definición de XBRL}

Nace de la propuesta realizada en 1998 por CPA Charles Hoffman, miembro de American Institute of Certified Public Accountants (AICPA), un experto contador y auditor, con la finalidad de simplificar la automatización del intercambio de información financiera mediante el uso del lenguaje XML.

En poco más de once años, esta sencilla idea ha conseguido atraer el interés y el respaldo de toda una comunidad de contadores y organizaciones, públicas y privadas, representando un gran número de países y coordinados en XBRL Internacional

La preparación de los reportes financieros tiene dos componentes principales: Los asistentes y el Proceso, en cada uno de dichos componentes se realizan las actividades de la Ilustración 2 Reporting Finaciero

Como se puede apreciar son muchas las actividades que deben de desarrollarse para generar y utilizar la información financiera, por lo que la principal ventaja de XBRL es que, a partir de un mismo origen y sin intervención de la empresa que difunde sus datos, diferentes usuarios obtienen los documentos que precisan con el formato e idioma que le es propio; con ello se elimina la necesidad actual de contestar a varios cuestionarios, para cumplir con los requerimientos específicos de cada demandante.

Cuando se prepara información manual, se debe identificar en forma visual muchos de los componentes de los estados financieros, tal como se muestra en la Ilustración 3 Balance Sheet, donde se aprecia que se debe de consignar información que es relevante en el análisis y sobre todo cuando se desea comparar información similar con otras empresas. Datos como: la entidad, periodo de tiempo, subentidad o división, conceptos que son mostrados, la moneda en que se expresa el reporte, las relaciones matemáticas que existen en dicho reporte, firmas y autorización y finalmente si están auditados. Toda esta información es consignada en los reportes pero no son fácilmente identificados por los sistemas automatizados.

\section{Taxonomias XBRL}

Las Taxonomías XBRL son los diccionarios del lenguaje XBRL. Consisten en esquemas de clasificación que definen etiquetas específicas para cada elemento específico de información (por ejemplo, «Beneficio Neto»).

Cada país tiene su propia Normativa Contable, por lo que cada una puede tener su propia Taxonomía para Informes Financieros. Es importante señalar que los usuarios de XBRL pueden ignorar en su totalidad, o al menos en gran parte, los detalles técnicos relativos al lenguaje. En cambio, las compañías desarrolladoras de software, tales como las que venden paquetes contables, deberían tener muy presente el XBRL y sus características en el diseño de sus productos.

Las taxonomías estarán adaptadas a las Normas Internacionales de Contabilidad (NIC). La adopción de este estándar por parte de las empresas no implicaría cambios en las normas de contabilidad, ya que se basa en los estándares existentes.

La presentación del XBRL constituye un metalenguaje o metadato que se representa como: $<$ concepto-a-representar $>$ Dato $</$ concepto-a- 
representar> a manera de ejemplo si queremos representar la utilidad de un Balance General, se presentará como:

$<$ Beneficios $>5000</$ Beneficios $>$.

Donde :

$<$ Beneficios $>\quad$ Representa la etiqueta de Inicio

$5000 \quad$ Representa al Dato a comunicar

$</$ Beneficios $>$ Representa la etiqueta final

XBRL es un estándar libre de royalties con lo que todos se pueden beneficiar de esta tecnología. Esto permite que el costo de implantación sea muy reducido. El costo inicial es el de la programación para adaptar el software, por la formación y las infraestructuras de desarrollo, mientras que el retorno de la inversión es muy rápido y, ayuda a elevar la productividad de las empresas.

Las principales características del XBRL son:

- Transparencia,

- flexibilidad,

- integridad y

- rapidez

Una vez implantado este estándar se podrían enviar los datos al otro lado del mundo y una vez recibidos tratarlos inmediatamente con lo que se consigue que la información sea actual y sea transmitida en tiempo real. Esto permite que el flujo de información sea más eficiente.

Las taxonomias pueden ser utilizadas en el intercambio de información financiera entre las empresas y los agentes que les demandan información, con varios fines (supervisores, estadísticos y otros).

Si se utiliza el formato XBRL para mantener la información de base en la computadora de quien emite los datos, un demandante al que se diera acceso podría seleccionar automáticamente la información requerida, porque localizaría con facilidad todos los elementos de su interés. Esto se consigue porque cada uno de los conceptos tiene una etiqueta aparejada, que lo define de forma inequívoca entre quienes utilizan el estándar XBRL. Así, por ejemplo, cada elemento incluido en unas cuentas anuales tiene asignada una etiqueta estándar, que puede ser leída e interpretada por la computadora del demandante de los datos. Las etiquetas se agrupan en taxonomías, o diccionarios de términos que contienen todos los elementos que se desean intercambiar. Las taxonomías son modulares, lo que quiere decir que pueden agruparse para facilitar posteriormente su utilización, y son extensibles, lo que significa que pueden compartir elementos comunes, mediante la definición de un núcleo común (o taxonomía "padre») que se extiende para cubrir necesidades específicas.

Por ejemplo, una empresa del sector eléctrico podría utilizar la taxonomía general del Plan General de Contabilidad, y a partir de ella, definir las partidas necesarias en las cuentas anuales específicas, en relación con la actividad que desarrollan. Las adaptaciones sectoriales serían ejemplos de extensiones nacionales de la taxonomía principal troncal.

La implantación como estándar de XBRL beneficia a:

- Los que generan la información,

- A los intermediarios que participan en su preparación y distribución,

- Los usuarios que la manejan.

Este lenguaje permite identificar los datos y facilita los cambios de reporting a lo largo del tiempo. Su flexibilidad es aplicable tanto entre los supervisores como entre países si logra convertirse en un estándar internacional. La información puede ser enviada y recibida desde diferentes fuentes y formatos, independientemente de la plataforma tecnológica y aplicaciones de software que se utilicen.

\section{DEFINICIÓN DE UN ELEMENTO CONTABLE MEDIANTE XBRL}

Su utilización por las empresas informantes se hace por medio de la generación de un informe XBRL, que, además de contener las características antes referidas, debe expresar la dimensión temporal (período de referencia) y espacial (si la unidad informante es individual o consolidada, por ejemplo) en un campo de «contexto» creado con esa finalidad.

El usuario de la información puede obtener finalmente un determinado formato de presentación, aplicando una hoja de estilo al informe XBRL. XBRL técnicamente es un esquema XML + Un conjunto de 5 linkbases: Presentación, Etiquetas, Cálculo, Definiciones y Referencias.

El consorcio XBRL Internacional, es el encargado del desarrollo del lenguaje informático XBRL, de la aprobación y publicación internacional de las taxonomías que se crean y del mantenimiento del estándar. El consorcio tiene una estructura descentralizada, por países, para favorecer el desarrollo de las taxonomías.

Existe una jurisdicción para una institución supranacional, dada su importancia como entidad reguladora contable, el IASB. La nómina de los países 
que participan en XBRL da una idea de la importancia de las acciones que se están desarrollando en Europa para el uso de este estándar en el intercambio de información financiera.

A continuación se muestra un modelo de una taxonomía con la finalidad de preparar balance general

\section{Taxonomías internacionales}

- DGI: Taxonomía para el reporting de los datos generales de identificación de agentes económicos.

- ES-BE-FS: La taxonomía ES-BE-FS ha sido diseñada para facilitar el envío de la información financiera contenida en los Estados Públicos Individuales y Consolidados, regulados por la Circular 4/2004. Esta información es transmitida periódicamente por las Entidades de Crédito a Banco de España.

- IPP: Taxonomía de la Comisión Nacional del Mercado de Valores para la elaboración de los modelos de información pública periódica de las entidades emisoras de valores admitidos a negociación en Bolsas de Valores según la circular $1 / 2005$.

- PGC90: Esta taxonomía permite el reporting electrónico de los datos económicos, financieros referentes a las empresas y particulares españoles obligados a realizar contabilidad de acuerdo con el Plan General de Contabilidad de 1990, y a su correspondiente depósito registral.

- ES-BE-CB: Esta taxonomía es utilizada por las empresas no financieras españolas que voluntariamente envían información económicofinanciera a la Central de Balances. La taxonomía se basa en los formatos normal y reducido que se envían anualmente a la Central de Balances.

- LENLOC: Taxonomía para la presentación telemática de la liquidación de los presupuestos de las entidades locales y de la información adicional requerida para la aplicación efectiva del principio de transparencia en el ámbito de la estabilidad presupuestaria.

- ES-BE-COREP: Esta taxonomía será utilizada por todas aquellas entidades de crédito que necesariamente tengan que reportar sus estados de solvencia de acuerdo al nuevo marco de capital Basilea II.

- PGC2007: El objeto de la taxonomía es el de facilitar a las empresas obligadas a depositar sus cuentas anuales individuales en el Registro Mercantil, el tratamiento contable

\begin{tabular}{|c|c|c|c|c|}
\hline Balance Sheet & iascf-pfs:BalanceSheet & Balance de situación & IAS 17 a & 2 \\
\hline Assets & lascf-pfs:Assets & Activos & IAS - CP & 3 \\
\hline Non Current Assets & lascf-pfs:NonCurrentAssets & Activos no corrientes & IAS 153 & 4 \\
\hline Property, Plant and Equipment & iascf-pfs:PropertyPlantEquipment & Inmovilizado material & IAS 166 a & 5 \\
\hline Investment Property & iascf-pfs:InvestmentProperty & Inmuebles de inversión & IAS $4069 \mathrm{c}$ & $\begin{array}{l}1 \\
4\end{array}$ \\
\hline Intangible Assets & iascf-pfs:IntangibleAssets & Activos inmateriales & IAS $166 \mathrm{~b}$ & $\begin{array}{c}1 \\
5\end{array}$ \\
\hline Biological Assets & iascf-pfs:BiologicalAssets & Activos biológicos & IAS 4139 & $\begin{array}{l}2 \\
3\end{array}$ \\
\hline Investments in Subsidiaries & iascf-pfs:InvestmentsSubsidiaries & $\begin{array}{l}\text { Inversiones en empresas } \\
\text { dependientes }\end{array}$ & IAS $2729 ;$ IAS 2730 & $\begin{array}{l}2 \\
4\end{array}$ \\
\hline Investments in Associates & iascf-pfs:InvestmentsAssociates & $\begin{array}{l}\text { Inversiones en empresas } \\
\text { asociadas }\end{array}$ & IAS 166 d; IAS 2828 & $\begin{array}{r}2 \\
5 \\
\end{array}$ \\
\hline Investments in Joint Ventures & iascf-pfs:InvestmentsJointVentures & Inversiones en negocios conjuntos & IAS $166 \mathrm{~d}$; IAS 3147 & $\frac{2}{6}$ \\
\hline Deferred Tax Assets & iascf-pfs:DeferredTaxAssets & Activos por impuestos diferidos & IAS 12 70; IAS 12 69; IAS $166 \mathrm{i}$ & $\begin{array}{l}2 \\
7\end{array}$ \\
\hline Other Financial Assets, Non Current & iascf-pfs:OtherFinancialAssetsNonCurrent & $\begin{array}{l}\text { Otros activos financieros, no } \\
\text { corrientes }\end{array}$ & IAS $166 \mathrm{c}$ & $\begin{array}{l}2 \\
8\end{array}$ \\
\hline Hedging Instruments, Non Current & iascf-pfs:NonCurrentAssetsHedgingInstrumentsNonCurrent & $\begin{array}{l}\text { Instrumentos de cobertura, no } \\
\text { corrientes }\end{array}$ & IAS $166 \mathrm{c}$ & $\begin{array}{l}3 \\
4 \\
4\end{array}$ \\
\hline $\begin{array}{l}\text { Trade and Other Receivables, Non } \\
\text { Current }\end{array}$ & iascf-pfs: TradeOtherReceivablesNonCurrent & $\begin{array}{l}\text { Deudores comerciales y otras } \\
\text { cuentas por cobrar, no corrientes }\end{array}$ & IAS $166 \mathrm{f}$ & $\begin{array}{l}3 \\
8 \\
\end{array}$ \\
\hline Prepayments, Non Current & iascf-pfs:PrepaymentsNonCurrent & Anticipos, no corrientes & IAS $173 \mathrm{~b}$ & $\begin{array}{l}4 \\
3\end{array}$ \\
\hline Cash Restricted or Pledged & iascf-pfs:CashRestrictedPledged & Caja restringida o pignorada & IAS 157 & $\begin{array}{l}4 \\
4\end{array}$ \\
\hline Other Assets, Non Current & iascf-pfs:OtherAssetsNonCurrent & Otros activos, no corrientes & IAS - CP & $\begin{array}{l}4 \\
5 \\
\end{array}$ \\
\hline Current Assets & lascf-pfs:CurrentAssets & Activos corrientes & IAS 153 & 4 \\
\hline $\begin{array}{l}\text { Property Plant and Equipment Held for } \\
\text { Sale, Current }\end{array}$ & iascf-pfs:PropertyPlantEquipmentHeldSaleCurrent & $\begin{array}{l}\text { Inmovilizado material mantenido } \\
\text { para ser enajenado, corriente }\end{array}$ & IAS $1666 \mathrm{c}$ & 4 \\
\hline Construction in Progress, Current & iascf-pfs:ConstructionProgressCurrent & $\begin{array}{l}\text { Inmovilizado material en curso de } \\
\text { construcción, corriente }\end{array}$ & IAS $1661 \mathrm{c}$ & $\begin{array}{l}4 \\
8 \\
\end{array}$ \\
\hline Inventories & iascf-pfs:Inventories & Existencias & IAS $234 \mathrm{~b}$; IAS $166 \mathrm{e}$ & $\begin{array}{l}4 \\
9\end{array}$ \\
\hline Other Financial Assets, Current & iascf-pfs:OtherFinancialAssetsCurrent & $\begin{array}{l}\text { Otros activos financieros, } \\
\text { corrientes }\end{array}$ & IAS $166 \mathrm{c}$ & $\begin{array}{l}5 \\
6 \\
\end{array}$ \\
\hline Hedging Instruments, Current & iascf-pfs:CurrentAssetsHedginglnstrumentsCurrent & $\begin{array}{l}\text { Instrumentos de cobertura, } \\
\text { corrientes }\end{array}$ & IAS $166 \mathrm{c}$ & $\begin{array}{l}6 \\
2 \\
2\end{array}$ \\
\hline Current Tax Receivables & iascf-pfs:CurrentTaxReceivables & Impuestos corrientes a cobrar & IAS 166 i; IAS 1269 & $\begin{array}{l}6 \\
6 \\
\end{array}$ \\
\hline Trade and Other Receivables, Current & iascf-pfs:TradeOtherReceivablesCurrent & $\begin{array}{l}\text { Deudores comerciales y otras } \\
\text { cuentas por cobrar, corrientes }\end{array}$ & IAS $166 \mathrm{f}$ & $\begin{array}{l}6 \\
7 \\
\end{array}$ \\
\hline Prepayments, Current & iascf-pfs:PrepaymentsCurrent & Anticipos, corrientes & IAS - CP & 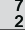 \\
\hline Cash and Cash Equivalents & iascf-pfs:CashCashEquivalents & $\begin{array}{l}\text { Efectivo y otros medios liquidos } \\
\text { equivalentes }\end{array}$ & IAS $166 \mathrm{~g}$ & $\begin{array}{l}7 \\
3 \\
\end{array}$ \\
\hline Other Assets, Current & iascf-pfs:OtherAssetsCurrent & Otros activos, corrientes & IAS - CP & 78 \\
\hline
\end{tabular}


de la información que han de suministrar los documentos que integran las mismas, para que el resultado final coincida con los modelos de depósito de cuentas en el Registro Mercantil. Asimismo, se trata de facilitar la presentación de magnitudes contables y financieras que sean necesarias para cualquier tratamiento de la información contenida.

- ICAL90: La Taxonomía ICAL90 es el instrumento tecnológico en virtud del cual la Cámara de Cuentas de Andalucía pone a disposición pública de la ciudadanía la información contable histórica de las entidades locales y empresas relacionadas en XBRL, el formato óptimo para su procesamiento digital y pieza clave para dar soporte a la toma de decisiones de los agentes económicos y sociales del siglo XXI.

- RSC: La Taxonomía RSC da soporte tecnológico a la generación, transmisión y procesamiento de Informes sobre actividades y situación de Responsabilidad Social Corporativa de empresas y todo tipo de entidades.

\section{Canales de Transferencia de Datos empleados en el Perú}

Hemos obtenido una muestra de tres entidades que vienen recibiendo información financiera de las empresas supervisadas:

1. El Banco Central de Reserva del Perú

2. El Conasev

3. La Superintendencia de Banca, Seguros y AFP

Con la finalidad de obtener, almacenar y analizar la información recibida cada entidad cuenta con el siguiente software:

1. Sistema de Liquidación Bruta en Tiempo Real, (Sistema LBTR).- Establecido por el Banco Central de Reserva, a través del que se liquidan las transacciones entre los agentes del sistema financiero, de tal forma que se convierte en el núcleo del sistema de pagos.

2. Sistema MVNet.- Es un sistema electrónico de intercambio de información, basado en tecnología PKI, que utilizando la tecnología de las firmas digitales e Internet, permite el intercambio de información seguro y eficiente, entre las entidades obligadas y CONASEV.

3. Submódulo de Captura y Validación Externa (SUCAVE).- Aplicable a las empresas del sistema financiero y del sistema de seguros, formulado por la Superintendencia de Banca, Seguros y AFP.
Del análisis preliminar llevado a cabo en cada uno de las entidades, hemos determinado la existencia de las situaciones siguientes:

1. Las entidades reguladoras mencionadas, solicitan la misma información, pero en diferentes formatos.

2. En cada empresa debe asignarse analistas dedicados a tiempo completo a preparar reportes para cada una de las entidades reguladoras.

3. Las entidades reguladoras solicitan modificaciones en los reportes que requieren tiempo para ser realizadas, ya que deben atender a mas de una en algunos casos

4. Las entidades reguladoras tienen sistemas de diferentes tecnologías

5. Los conceptos tienen diferentes niveles de detalle o de definición, según cada unidad reguladora.

6. La misma información se envía en diferentes formatos y tecnologías lo que impide la estandarización de los reportes.

7. Los analistas deben «copiar y pegar» desde diferentes fuentes de información para obtener una información integral de la situación de las empresas.

8. Las entidades reguladoras publican la información en diferentes formatos y medios

9. Las estadísticas difieren en función al nivel de detalle definido por cada regulador.

\section{CONCLUSIONES}

1. El XBRL será el lenguaje estándar para el reporte de información financiera. Los estándares internacionales pueden ser incorporados a las taxonomías de XBRL. El XBRL es un lenguaje informático, pero la taxonomía corresponde a los usuarios del negocio.

2. El estándar XBRL responde a las exigencias del mercado, porque ofrece el soporte necesario para conseguir que los datos sean transmitidos de forma rápida, segura y transparente, que puedan ser consultados y comparados por cualquier empresa y en cualquier país. Esta facilidad no sólo es a nivel externo, también incide en la estructura interna de la empresa ya que agiliza el proceso a la hora de generar y publicar informes.

3. La integridad de la información es inherente al estándar XBRL, ya que a cada dato se le aplica una etiqueta que permanece siempre. Además dicha etiqueta sólo es generada una sola vez, permitiendo su reutilización, lo que hace que sea más fiable, se reduzcan los errores humanos y, en consecuencia, los costes. 
4. Debe existir un sólo requerimiento de información basado en taxonomías consistentes e integradas. Debe ser fácil de obtener la información para el usuario final (interno y externo): extraer, consumir, analizar, comparar, publicar.

\section{RECOMENDACIONES}

1. Es importante que todas las entidades que se verán beneficiadas de la implementación de XBRL en el Perú (Universidades, Empresas Públicas y Privadas) formen un grupo sólido donde se apoyen mutuamente, compartiendo sus conocimientos, recursos económicos, desarrollos de software, formando grupos de investigación entre las entidades partícipes esto con el fin de que los beneficios que traería XBRL al intercambio de información financiera, ya sea tanto nivel nacional como internacional, se obtengan para Perú a la velocidad que un mundo globalizado y con soluciones tecnológicas a los negocios de comercio de bienes y servicios está ofreciendo.

2. Incentivar a los alumnos de la Universidad para que presenten proyectos de investigación, sobre el diseño de taxonomía de XBRL, para los diversos Sectores económicos del país. Propiciar la creación de un prototipo de software funcional basado en XBRL.

3. Difusión de material didáctico, bibliográfico recopilado en manuales, tutoriales y documentos relacionados al tema para ser discutidos en clase.

4. Creación de la plataforma virtual para la enseñanza del estándar XBRL, sus diversos usos, aplicaciones, diseño de taxonomías entre otros.

\section{REFERENCIAS BIBIOGRÁFICAS}

1. Roger Debreceny, Carsten Felden, Bartosz Ochocki, Maciej Piechocki, XBRL for Interactive Data: Engineering the Information Value Chain (Hardcover), Springer,2009,p. 213

2. BCRP (2008): "Sistema LRBT". [Fecha de consulta: 07/09/09]. Accesible desde http:// www.bcrp.gob.pe/sistema-de-pagos/sistema$\underline{\text { lbtr.html. }}$

3. CONASEV (2008): "Sistema MVNet". [Fecha de consulta: 07/09/09]. Accesible desde http:// www.bcrp.gob.pe/sistema-de-pagos/sistema$\underline{\text { lbtr.html. }}$
4. SBS (2009): «Sistema Sucave. [Fecha de consulta: 07/09/09]. Accesible desde http://www.sbs. gob.pe/PortalSBS/Sucave/index.htm. 